\title{
High temperature magnetic properties of nanocrystalline $\mathrm{Sn}_{0.95} \mathrm{Co}_{0.05} \mathrm{O}_{2}$
}

\author{
O MOUNKACHI ${ }^{1}$, E SALMANI $^{2}$, M BOUJNAH $^{2}$, H LABRIM $^{3}$, H EL MOUSSAOUI ${ }^{1,2}$, \\ M HAMEDOUN $^{1, *}$, A BENYOUSSEF ${ }^{1,2}$, A EL KENZ $^{2}$, H EZ-ZAHRAOUY $^{2}$, R MASROUR $^{4}$ and \\ E K HLIL ${ }^{5}$ \\ ${ }^{1}$ The Institute for Nanomaterials and Nanotechnology, MAScIR (Moroccan Foundation for Advanced Science, \\ Innovation and Research), Rabat, Morocco \\ ${ }^{2}$ LMPHE (URAC 12), Departement of Physique, BP 1014, Faculty of Science, Mohammed V-Agdal University, \\ Rabat, Morocco \\ ${ }^{3}$ National Centre for Energy, Sciences and Nuclear Techniques, CNESTEN, Morocco \\ ${ }^{4}$ Laboratory of Materials, Processes, Environment and Quality, Cady Ayyed University, \\ National School of Applied Sciences, Safi, Morocco \\ ${ }^{5}$ Institut Néel, CNRS et Université Joseph Fourier, BP 166, F-38042 Grenoble Cedex 9, France
}

MS received 17 October 2012; revised 17 December 2012

\begin{abstract}
Structural and magnetic properties of $\mathrm{Sn}_{0.95} \mathrm{Co}_{0.05} \mathrm{O}_{2}$ nanocrystalline and diluted magnetic semiconductors have been investigated. This sample has been synthesized by co-precipitation route. Study of magnetization hysteresis loop measurements infer that the sample of $\mathrm{Sn}_{0.95} \mathrm{Co}_{0.05} \mathrm{O}_{2}$ nanoparticle shows a well-defined hysteresis loop at $300 \mathrm{~K}$ temperature, which reflects its ferromagnetic behaviour. We confirmed the roomtemperature intrinsic ferromagnetic (FM) semiconductors by ab initio calculation, using the theory of the functional of density (DFT) by employing the method of Korringa-Kohn-Rostoker (KKR) as well as coherent potential approximation (CPA, explain the disorder effect) to systems. The ferromagnetic state energy was calculated and compared with the local-moment-disordered (LMD) state energy for local density approximation (LDA) and LDA-SIC approximation. Mechanism of hybridization and interaction between magnetic ions in $\mathrm{Sn}_{0.95} \mathrm{Co}_{0.05} \mathrm{O}_{2}$ is also investigated. To explain the origin of ferromagnetic behaviour, we give information about total and atoms projected density of state functions.
\end{abstract}

Keywords. Diluted magnetic semiconductors; ferromagnetic, $\mathrm{SnO}_{2}$; ab-initio calculation; KKR-CPA; spintronic.

\section{Introduction}

Synthesis of diluted magnetic semiconductors (DMS) obtained by doping magnetic transition elements such as $\mathrm{Mn}, \mathrm{Fe}$ and $\mathrm{Co}$ into nonmagnetic semiconductors is one of the most active topics in the spintronics field, which is considered as an alternative to the traditional semiconductor electronics (Ohno 1998; Prinz 1998). Study of spintronic materials has been stimulated by theoretical development, which has shown that wide-band-gap semiconductors are the most promising candidates for achieving high Curie temperatures $\left(T_{\mathrm{C}}\right)$ (Dielt et al 2000). One of the goals of recent intense research in the field of magnetic materials is to obtain room-temperature intrinsic ferromagnetic (FM) semiconductors. However, numerous studies performed on these systems have led to a controversy on the nature (intrinsic or extrinsic) of the ferromagnetism in these systems. These include Co-doped

*Author for correspondence (hamedoun@gmail.com)
$\mathrm{TiO}_{2}$ (Matsumoto et al 2001), Cr-doped AlN (Wu et al 2003), Fe-doped (Han et al 2002), V-doped (Saeki et al 2001) and Co-doped $\mathrm{ZnO}$ (Belghazi et al 2006). Among promising host materials for diluted magnetic semiconductor (DMS) systems, $\mathrm{SnO}_{2}$ have attracted considerable attention due to a number of unusual magneto-optical, electronic and magnetic properties, including a magnetically tunable bandgap, resulting from large $s p-d$ exchange interaction between the magnetic ions and the band electrons. Tin dioxide $\left(\mathrm{SnO}_{2}\right)$ is an $n$-type semiconductor with a crystalline structure of the rutile type. It is a semiconductor with wide-band-gap, around $3.6 \mathrm{eV}$ (Cho et al 2002; Fang et al 2008), and its optical and electrical properties are widely used in technological applications such as in solar cells, gas sensing (Hidalgo et al 2005), transparent electrodes (Lalauze et al 1987) and optoelectronic devices (Vishwakarma et al 1993). As we all know, doping can improve the performance of $\mathrm{SnO}_{2}$. Many results have been reported on $\mathrm{Fe}$ (Fitzgerald et al 2004; Punnoose et al 2005), Co (Hong et al 2005a; Hong and Sakai 2005; Srinivas et al 2009; Bagheri 2010), Ni 
(Hong et al 2005), Mn-doped (Wang et al 2008; Liu et al 2009) $\mathrm{SnO}_{2}$ oxides. Coey et al (2004) found ferromagnetic stability in Fe-doped $\mathrm{SnO}_{2}$ thin film with moments ranging from 1.06 to $4.76 \mu_{\mathrm{B}} / \mathrm{Fe}$. Many works and conflicting results for ferromagnetic properties of Co-doped $\mathrm{SnO}_{2}$, Ogale et al (2003) reported high temperature ferromagnetism with giant magnetic moment in transparent Co-doped $\mathrm{SnO}_{2-\delta}$, ferromagnetic properties at room temperature was observed by Fitzgerald et al (2006) for Co-doped thin. Nevertheless, ferromagnetism is much more difficult to find and understand (intrinsic or extrinsic). Experimentally, the prediction of high temperature ferromagnetism depends on many experimental parameters: preparation methods, measurement techniques, substrate chooses, unexpectedly carriers introduced during the synthesis, for example, $\mathrm{H}$ or $\mathrm{O}$ and acceptor or donor defects. Especially in Co-doped $\mathrm{SnO}_{2}$ polycrystalline sample compared to Co-doped thin film, a room-temperature ferromagnetism was observed in nanocrystalline $\mathrm{Sn}_{0.95}$ $\mathrm{Co}_{0.05} \mathrm{O}_{2}$-based diluted magnetic semiconductors less than $5 \%$ of Co (Srinivas et al 2009). The theoretical prediction of room temperature ferromagnetism in Co-doped $\mathrm{SnO}_{2}$ was made by Rahman et al (2008) and Zhang and Yan (2009) using ab-initio calculation with GGA or LDA approximation without SIC correction.

In the present study, Co-doped $\mathrm{SnO}_{2}$ nanocrystalline was synthesized by the co-precipitation method. The structure and gain sizes of the samples were evaluated using X-ray diffraction. Magnetic properties of the products were characterized with a superconducting quantum interference device (SQUID) to determine magnetization hysteresis and magnetization as a function of temperature. $\mathrm{ZFC} / \mathrm{FC}$ measurements were carried out in the temperature range of 5-360 K with an applied field of 500 Oe. To explain the mechanism of origin of ferromagnetism in Co-doped $\mathrm{SnO}_{2}$, we have performed $a b$ initio calculations using the KKR-CPA method. The ferromagnetic states (FM) energy was calculated and compared with the local-moment-disordered (LMD) state energy for LDA (local density approximation) and LDA-SIC approximation. Mechanism of hybridization and interaction between magnetic ions in $\mathrm{Sn}_{0 \cdot{ }_{5}} \mathrm{Co}_{0.05} \mathrm{O}_{2}$ are also investigated.

\section{Experimental}

\subsection{Experimental procedure}

The simple solution of $\mathrm{SnO}_{2}$ doped cobalt was prepared by a simple chemical co-precipitation method using $\mathrm{SnCl}_{2} \cdot 2 \mathrm{H}_{2} \mathrm{O}$ and $\mathrm{CoCl}_{2} \cdot 6 \mathrm{H}_{2} \mathrm{O}$, as the sources of $\mathrm{Sn}$ and $\mathrm{Co}$, respectively. The precursors were added in $\mathrm{H}_{2} \mathrm{O}$ and mixed homogeneously and refluxed under air atmosphere to yield a uniform mixture of precursor at $80^{\circ} \mathrm{C}$ for $30 \mathrm{~min}$, The $\mathrm{pH}$ of the solution was constantly monitored as the $\mathrm{NaOH}$ solution was added drop-wise. The dropping rate must be well controlled for the chemical homogeneity. The reactants were constantly stirred using a magnetic stirrer until a $\mathrm{pH}$ level of $>12$ was achieved. The precipitates were washed several times to remove the water-soluble impurities and free reactants and dried. The spongy contents were filtered, dried and then powdered. Heating treatments of the synthesized powders were realized at $800{ }^{\circ} \mathrm{C}$ for $8 \mathrm{~h}$. The crystal structure and particle size of the samples were determined using a Bruker-AXS D8 ADVANCE X-ray diffractometer (XRD) with $\mathrm{CuK} \alpha$ radiation $(\lambda=0.15406 \mathrm{~nm})$, while the magnetic characterization was done by magnetic properties measurement system (MPMS SQUID VSM Quantum design).

\section{$2.2 X$-ray diffraction pattern}

Figure 1 shows $\mathrm{X}$-ray pattern diffraction of Co-doped $\mathrm{SnO}_{2}\left(\mathrm{Sn}_{0.95} \mathrm{Co}_{0.05} \mathrm{O}_{2}\right)$. The positions and relative intensities of all the peaks indicate that the crystalline structure of the products favours the formation of rutile phase, which is accordant to JCPDS card No. 82-0514 for $\mathrm{SnO}_{2}$. The crystallite sizes of the produced $\mathrm{Sn}_{0 \cdot 95} \mathrm{Co}_{0.05} \mathrm{O}_{2}$ for the most intense peak (211) plane estimated from the X-ray diffraction data using the Debye-Scherrer formula:

$$
d_{\mathrm{RX}}=\frac{K \lambda}{\beta \cos \theta},
$$

where $d_{\mathrm{RX}}$ is the crystallite size, $k=0.9$ the correction factor to account for particle shapes, $\lambda=1.5406 \AA$ the wavelength of $\mathrm{Cu}, \beta$ the full width at half maximum (FWHM) of the most intense diffraction peak (211) plane and $\theta$ is the Bragg angle. The average value of the crystallites dimension for two samples was found to be $21 \cdot 14 \mathrm{~nm}$.

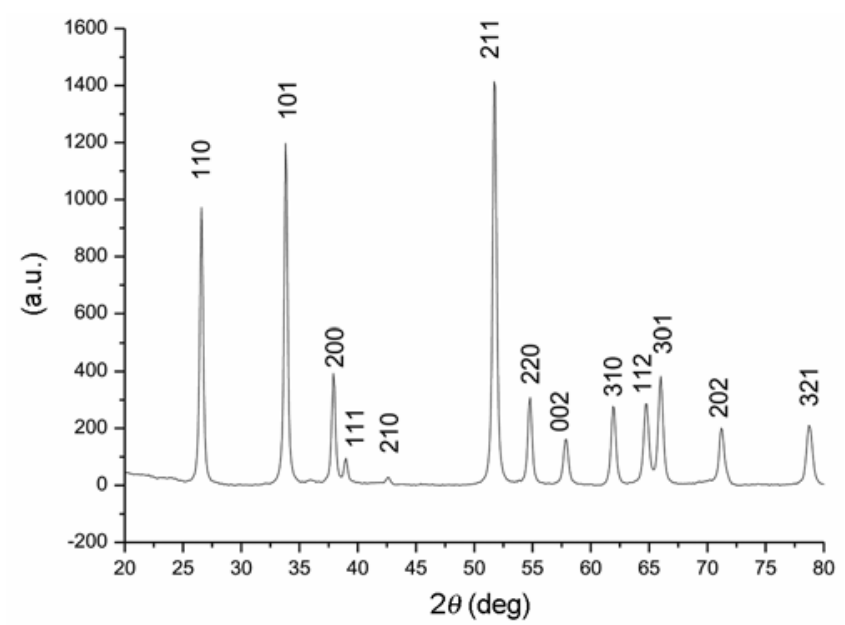

Figure 1. X-ray diffraction pattern for $\mathrm{Sn}_{0.95} \mathrm{Co}_{0.05} \mathrm{O}_{2}$ nanocrystalline. 


\subsection{Magnetic measurements}

The magnetic properties of $\mathrm{Sn}_{0.95} \mathrm{Co}_{0.05} \mathrm{O}_{2}$ nanocrystalline have been studied by measuring the magnetization as a function of temperature in applied field of $500 \mathrm{Oe}$. The zero field-cooled (ZFC) and field-cooled (FC) curves are shown in figure 2 , which have low magnetic anisotropy of $\mathrm{SnO}_{2}$-doped cobalt. The corresponding Curie temperature is higher than room temperature. Figure 3 shows magnetic field dependence of magnetic moment at $5 \mathrm{~K}$ and room temperature $(300 \mathrm{~K})$ of $\mathrm{Sn}_{0.95} \mathrm{Co}_{0.05} \mathrm{O}_{2}$. The hysteresis loops close at low field and the magnetization seems to approach saturation at $\sim 10 \mathrm{kOe}$, especially, at room temperature. The saturation moment is $\left(M_{\mathrm{s}}=\right.$ $\left.0.18 \mathrm{emu} / \mathrm{g} ; M_{\mathrm{s}}=0.095 \mu_{\mathrm{B}} / \mathrm{Co}\right)$ and the coercive field is $\left(H_{\mathrm{c}}=7 \mathrm{Oe}\right)$ measured at room temperature $(300 \mathrm{~K})$. Like this magnetic character was observed in same DMS nanoparticule in Co-doped $\mathrm{SnO}_{2}$ (Srinivas et al 2009)

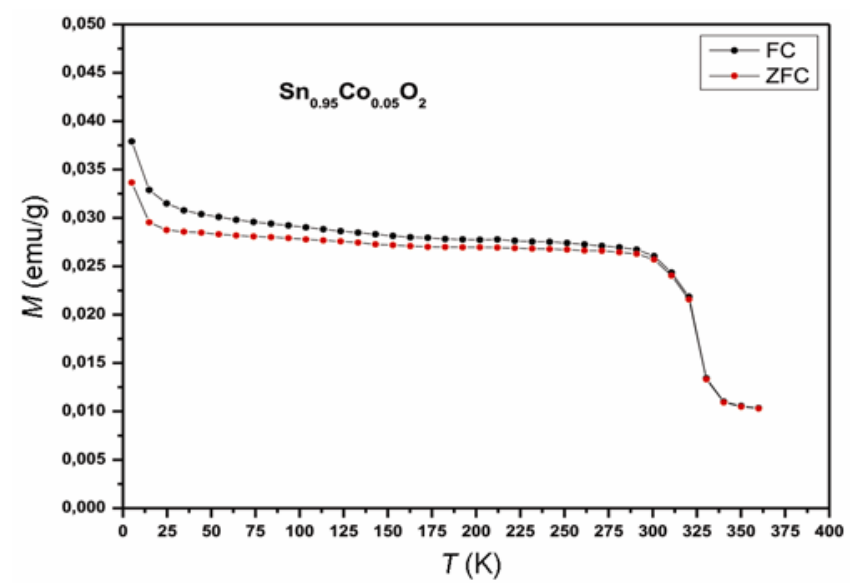

Figure 2. Magnetization vs temperature measured on a $\mathrm{Sn}_{0.95} \mathrm{Co}_{0.05} \mathrm{O}_{2}$ nanocrystalline at external magnetic field of 500 Oe.

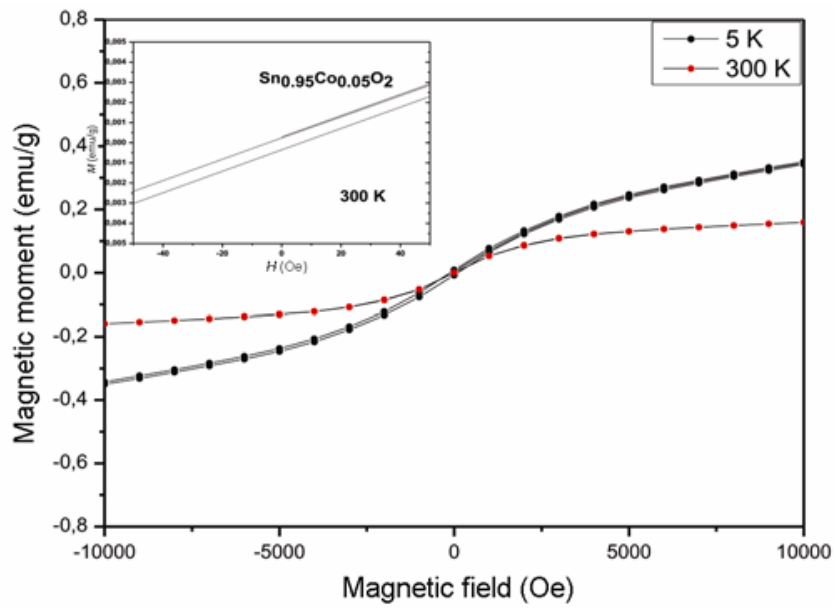

Figure 3. Magnetic hysteresis loops of $\mathrm{Sn}_{0.95} \mathrm{Co}_{0 \cdot 05} \mathrm{O}_{2}$ nanocrystalline at temperatures 5 and $300 \mathrm{~K}$. and Co-doped $\mathrm{ZnO}$ (Pal and Gir 2011) by simple doping method or by co-doping method (Mounkachi et al 2012). Indeed, figure 4 shows a plot of inverse d.c. magnetic susceptibility $(1 / \chi=\mathrm{H} / \mathrm{M})$ as a function of temperature for Co-doped $\mathrm{SnO}_{2}$. As can be seen from this figure, the plot follows a linear Curie-Weiss type behaviour, $\chi=\mathrm{C} /[T-\theta]$. The inverse d.c. magnetic susceptibility $(1 / \chi=\mathrm{H} / \mathrm{M})$ varies linearly with the temperature, following Curie-Weiss type behaviour, $\chi=C /[T-\theta]$. This means that the magnetic impurities (Co) are coupled ferromagnetically. The appearance of ferromagnetism in the pristine Co-doped $\mathrm{SnO}_{2}$ nanocrystalline may be attributed to effective exchange interactions between magnetic impurities (cobalt) or by unpaired electron spins originating from the surface defects such as oxygen vacancy clusters instead of single neutral oxygen vacancies

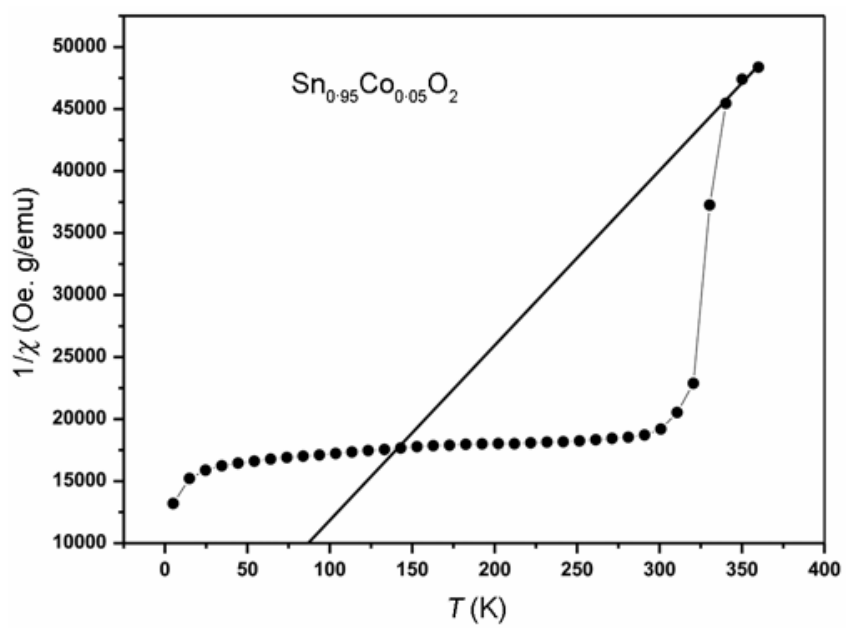

Figure 4. Inverse magnetic susceptibility vs temperature for $\mathrm{Sn}_{0.95} \mathrm{Co}_{0.05} \mathrm{O}_{2}$ nanocrystalline.

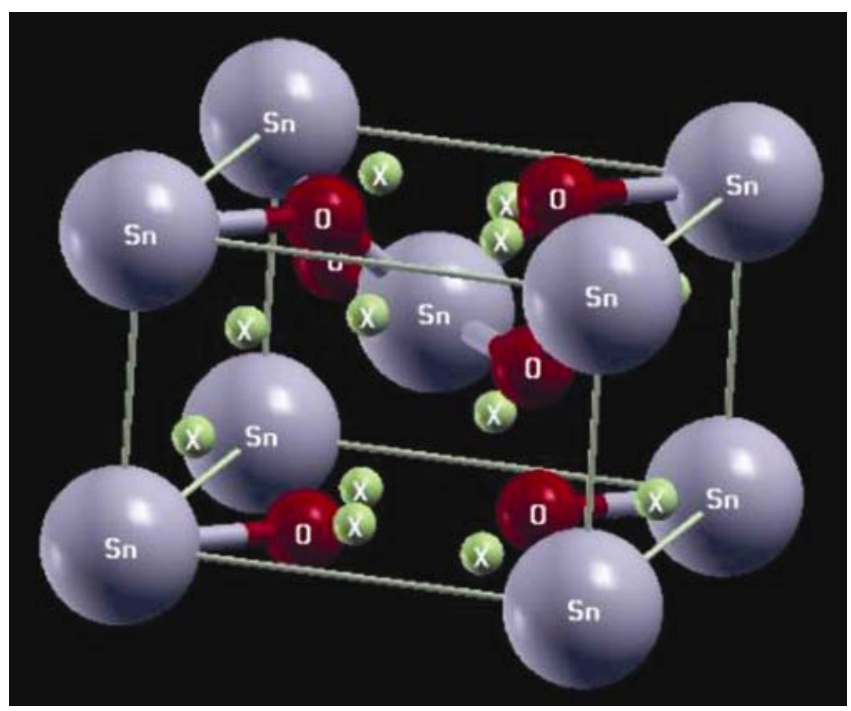

Figure 5. $\mathrm{SnO}_{2}$ in rutile structure. 

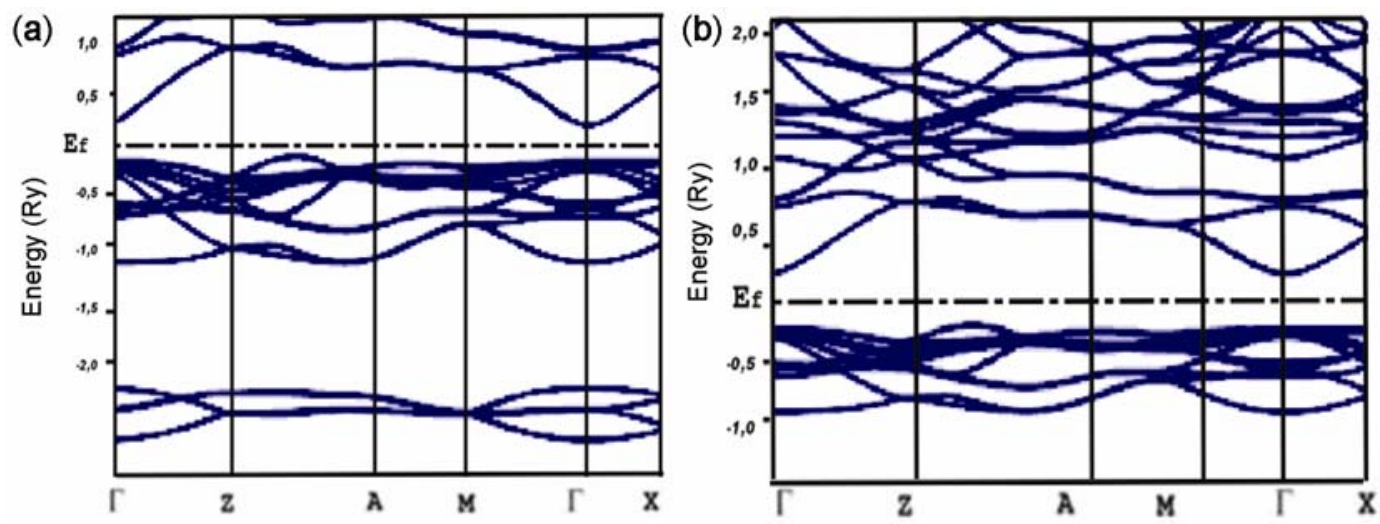

Figure 6. Band structure calculation of $\mathrm{SnO}_{2}$ with (a) LDA approximation and (b) LDA-SIC approximation.

or by secondary phases. In the case of Co-doped $\mathrm{SnO}_{2}$ samples, the origin of ferromagnetism is very complex. Although, different mechanisms might be responsible, in this case we use ab-initio calculation to understand this ferromagnetic stability. In our investigation, since the above structural characterizations have ruled out the presence of secondary phases including Co clusters, the observed ferromagnetism should be the intrinsic nature of the Co-doped $\mathrm{SnO}_{2}$ that we confirmed with electronic structure calculation.

\section{Theoretical investigation}

In the present work, the electronic structure has been calculated from $a b$-initio calculation, using the theory of the functional of density (DFT) by employing the method of Korringa-Kohn-Rostoker (KKR) as well as the coherent potential approximation (CPA, explain the disorder) to systems. CPA method is mean field theory and supposed to be effective medium which describes the configuration average of the electronic structure of a substitution alloy. The effective medium is calculated selfconsistently usually within the single site approximation. $\mathrm{SnO}_{2}$ has a tetragonal symmetry in rutile structure (figure $5)$. The rutile structure is characterized by two lattice parameters $a=4.73 \AA$ and $c=3.18 \AA$ computed from our experimental data. It is worth noting that for each atom the sphere radius is taken such that the volume of the unit cell is the sum of the volumes of the all atomic spheres. In order to achieve a good packing, we should add initially 'empty' spheres (ES) with $(Z=0)$ representing atomic inter-sites. Using the space group $P 4_{2} / m n m$ in the international tables of X-ray crystallography table $\mathrm{N}^{\circ} 136$, we put the six atoms, two tin atoms occupying 2 a Wyckoff positions: $(0,0,0)(1 / 2,1 / 2,1 / 2)$ and four oxygen ions occupying $4 f$ positions: $(u, u, 0)(-u,-u, 0)(1 / 2+u, 1 / 2-u$, $1 / 2)(1 / 2-u, 1 / 2+u, 1 / 2)$, while a set of eight ES has been localized at the positions: $(1 / 2,0,0 \cdot 1682)(0,1 / 2$, $-0.1682)(0,1 / 2,0.1682)(1 / 2,0,0.1682)(-0.3125$,
$0.3125,0)(-0 \cdot 1875,-0 \cdot 1875,0)(0 \cdot 1875,0 \cdot 1875,0)$, $(0.3125,-0.3125,0)$. This atomic configuration is presented in figure 5 .

In order to explain the mechanism of ferromagnetism in $\mathrm{Sn}_{0.95} \mathrm{Co}_{0.05} \mathrm{O}_{2}$, we have performed ab initio calculations using the KKR-CPA method, with the parameterization of Vosko (1980). Co impurities are introduced randomly into cation sites of the $\mathrm{SnO}_{2}$ semiconductor. To solve the DFT one-particle equations, we use multiple scattering theory, i.e. the KKR Green's function (KKRGF) method for the dilute impurity limit and the KKR coherent-potential approximation (KKR-CPA includes the disorder in calculations) for concentrated alloys. The form of the crystal potential is approximated by a muffintin potential, and the wave functions in the respective muffin-tin spheres were expanded in real harmonics up to $l=2$, where $l$ is the angular momentum quantum number defined at each site. We use higher K-points up to 456 in the irreducible part of the first Brillouin zone. In the present calculations, we used the KKR-CPA code MACHIKANEYAMA2008v08 package produced by Akai of Osaka University (Akai). In figures 6 (for band structure calculation) and 7 (for density of stat calculation), we show the band structure of bulk $\mathrm{SnO}_{2}$, a bandgap of $2.05 \mathrm{eV}$ for LDA approximation and $3.40 \mathrm{eV}$ for LDA-SIC approximation close to experimental value (Cho et al 2002; Fang et al 2008). As already known, $\mathrm{SnO}_{2}$ is a direct bandgap semiconductor $\left(E_{\Gamma-\Gamma}\right)$, with the lowest energy electron-hole transition occurring at the $\Gamma$ point. It is interesting to compare the present results with previous band structure calculations. The energy band structure of $\mathrm{SnO}_{2}$ consists of four bands. The low energy bands, between -1.2 and -1.6 Ry are composed mainly of the semi-core Sn-4d states, the next band is the wide valence band within the energy interval from -0.8 to $0 \cdot 0$ Ry consists of the hybridized $\mathrm{O}-2 p \mathrm{Sn}-5 p$ states and has three sub-bands. The conduction band which is higher on energy in the electron spectrum is built of the $5 s$ states of tin, which are typical for $\mathrm{SnO}_{2}$ as well as with the results of past calculations. 

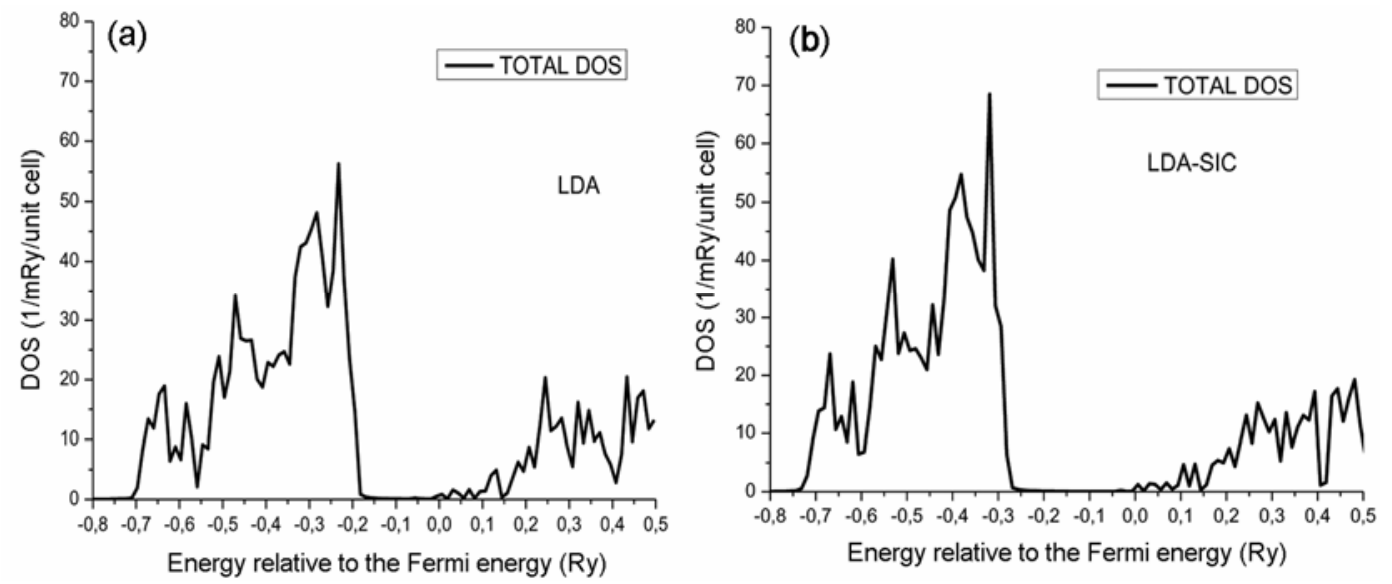

Figure 7. Total density of states of $\mathrm{SnO}_{2}$ for: (a) LDA calculation and (b) LDA-SIC approximation.

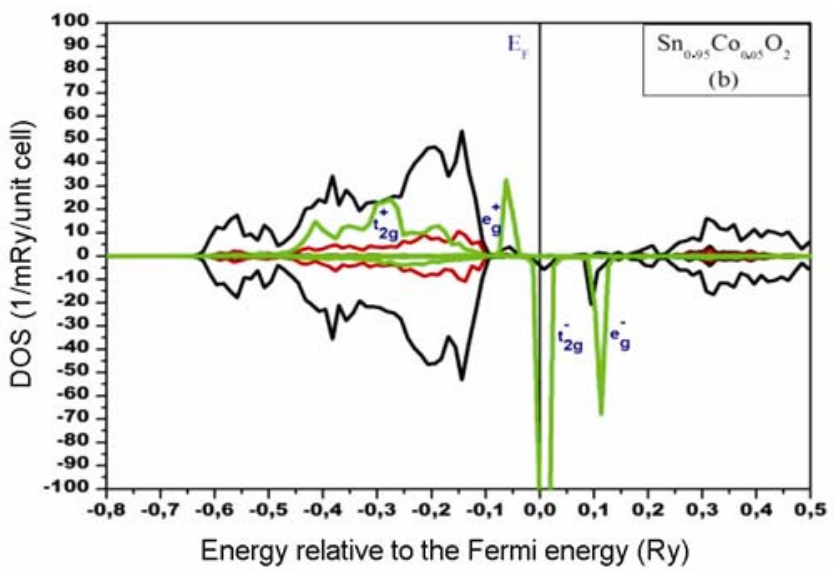

Figure 8. Total and partial density of states of ferromagnetic $\mathrm{Sn}_{0.95} \mathrm{Co}_{0.05} \mathrm{O}_{2}$ for LDA calculation.

Table 1. Calculated magnetic moments (total and partial) in $\mathrm{Sn}_{0.95} \mathrm{Co}_{0.05} \mathrm{O}_{2}$ and energy difference between spin glass and ferromagnetic state.

\begin{tabular}{lcccc}
\hline Co-doped $\mathrm{SnO}_{2}$ & $M\left(\mu_{\beta}\right)$ & $M^{\mathrm{Co}}\left(\mu_{\beta}\right)$ & $M^{\mathrm{O}}\left(\mu_{\beta}\right)$ & $\Delta E(\mathrm{meV})$ \\
\hline LDA & $0 \cdot 20$ & 3.54 & 0.01 & 5.26 \\
LDA-SIC & 0.18 & 3.39 & 0.00 & 5.08
\end{tabular}

We study the magnetic properties of $\mathrm{SnO}_{2}$, doped in $\mathrm{Sn}$ positions with $5 \%$ of single transition elements TM, which are $\mathrm{Co}$, in order to compare with our experimental observations. Doping with $5 \%$ of Co ion can change the magnetic property of $\mathrm{SnO}_{2}$. The result of magnetism in $\mathrm{Sn}_{0.95} \mathrm{Co}_{0.05} \mathrm{O}_{2}$ is illustrate in table 1 , which shows that the Co-doped $\mathrm{SnO}_{2}$ have magnetic moments which are $3.54 \mu_{\mathrm{B}}$ with LDA, but when we add the SIC correction the magnetic moments are $3.39 \mu_{\mathrm{B}}$. However, the magnetic moments per cobalt ion, calculated from the magnetization data is $M_{\mathrm{s}}=0.095 \mu_{\mathrm{B}} / \mathrm{Co}$ for $300 \mathrm{~K}$ and $M_{\mathrm{s}}=0.18 \mu_{\mathrm{B}} /$ Co for $5 \mathrm{~K}$, for low temperature comparable to the theoretical calculation (table 1).

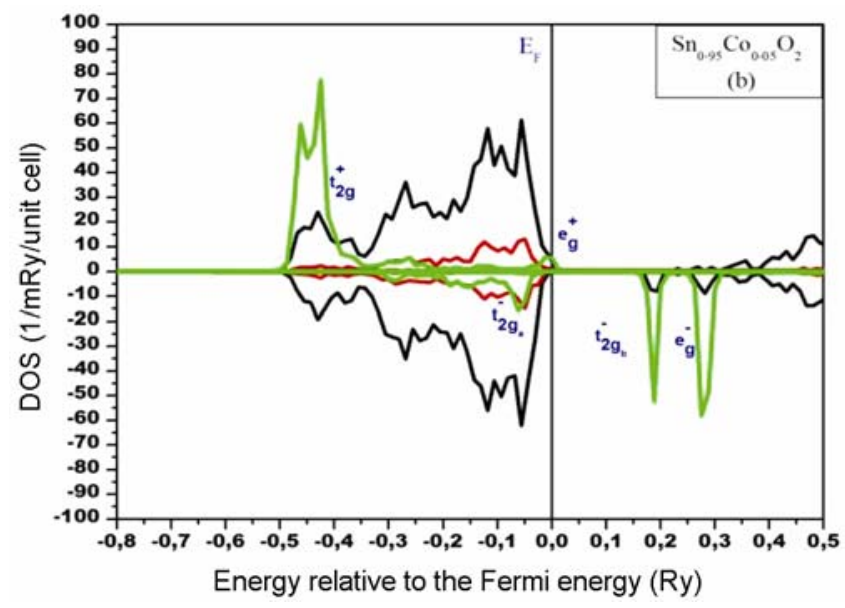

Figure 9. Total and partial density of states of ferromagnetic $\mathrm{Sn}_{0.95} \mathrm{Co}_{0.05} \mathrm{O}_{2}$ for LDA-SIC calculation.

In order to determine the most stable magnetic state, the energy difference between the

$$
\Delta E=E(\text { spin-glass state })-E(\text { ferromagnetic state }),
$$

ferromagnetic state (FM) and disordered local moment (DLM) have been calculated. DLM state describes the spin-glass state at finite temperature where the directions of all local moments are randomly distributed, so that the average magnetization vanishes. The variation of the $\Delta E$ as a function of LDA approximations (LDA and LDASIC) are given in table 1 , in which it is shown that $\Delta E$ has always positive values with LDA and LDA-SIC. These indicate that the ferromagnetic state is more stable than the spin glass for both cases, and it is correlated with our and author experimental results (Ogale et al 2003; Coey et al 2004; Srinivas et al 2009) and with theoretical results (Wang et al 2007, 2008). In order to understand and to explain the more stabilized ferromagnetism state in $\mathrm{Sn}_{0.95} \mathrm{Co}_{0.05} \mathrm{O}_{2}$, the density of states (DOS) of this system for LDA without and within SIC was calculated and they are presented in figures 8 and 9 within LDA and 

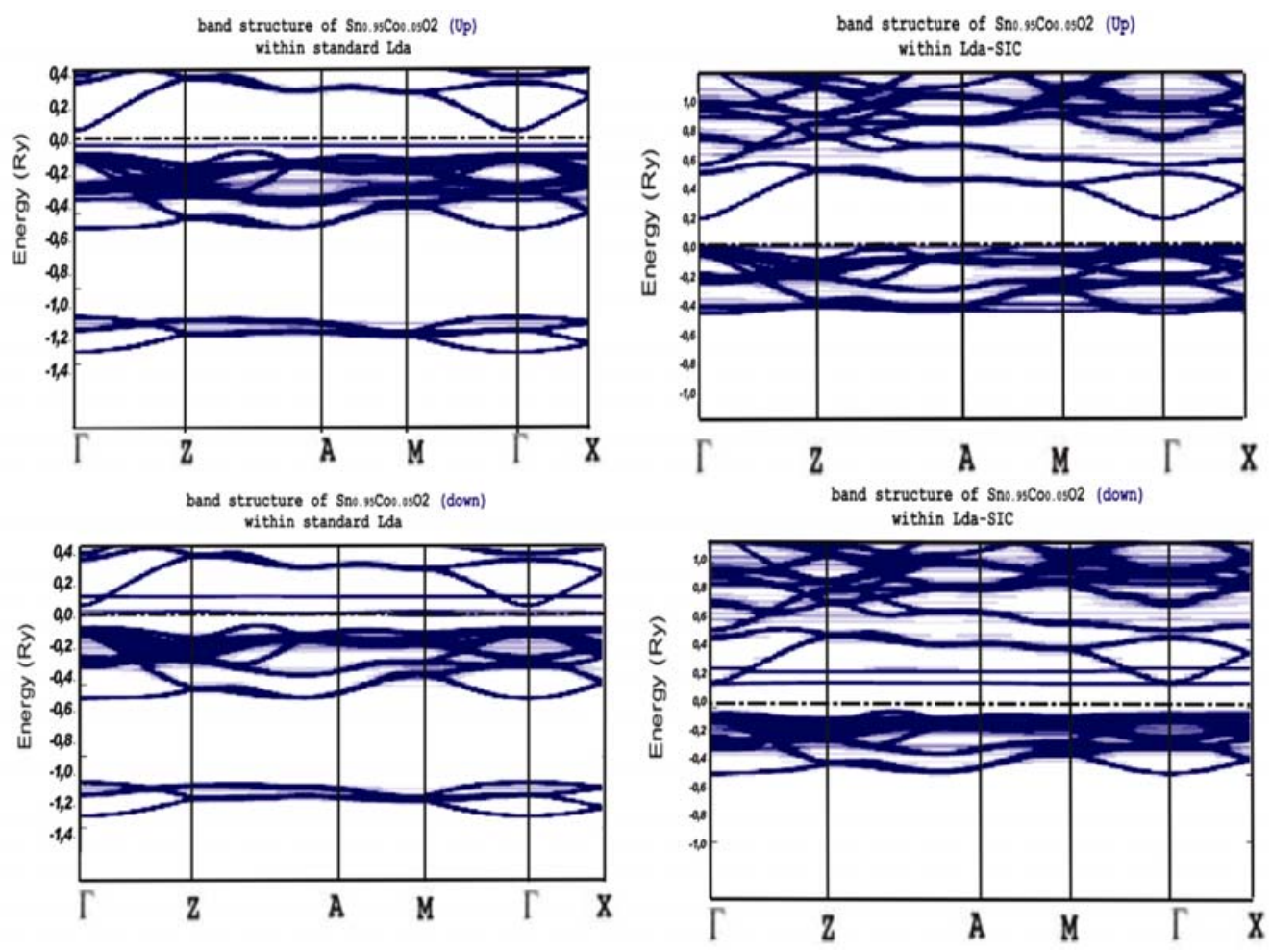

Figure 10. Band structures calculation for spin-up and spin-down of $\mathrm{Sn}_{0 \cdot 95} \mathrm{Co}_{0.05} \mathrm{O}_{2}$ within LDA and LDA-SIC approximation, in comparison with pure $\mathrm{SnO}_{2}$.

LDA-SIC, respectively. In Co ion-doped rutile $\mathrm{SnO}_{2}, \mathrm{O}$ ions around Co ions form an octahedral crystal field, splitting $d$ orbitals into lower $t_{2 \mathrm{~g}}$ states and upper $e_{\mathrm{g}}$ states as indicated in figure 8 (for LDA approximation). The five electrons in $3 d$ are occupied with 3 spins up in $t_{2 \mathrm{~g}}$ state and 2 spin up in $e_{\mathrm{g}}$ state, the exchange splitting between $t_{2 \mathrm{~g}}$ states is larger than the crystal field spitting between $t_{2 \mathrm{~g}}$ and $e_{\mathrm{g}}$ states in Co-doped $\mathrm{SnO}_{2}$. Besides from figure 8 , when we add SIC correction to LDA, we observed the modification of arrangement in electronic structure, such that we obtain 4 electrons in $t_{2 \mathrm{~g}}$ state instead of 3 electrons, with 3 is on spin up, is 1 on spin down, and the remaining 1 is on spin up in $e_{\mathrm{g}}$ state, which leads to Jahn-Teller effect. Indeed, after the rule of Jahn Teller effect, the $t_{2 \mathrm{~g}}$ states for down and up-spin side are leading to the formation of $t_{2 \mathrm{~g}}$ fractions, is indicate in figure 9. Figure 10 shows the band structures calculation for spin-up and spin-down of $\mathrm{Sn}_{0.95} \mathrm{Co}_{0.05} \mathrm{O}_{2}$ within LDA and LDA-SIC approximation, in comparison with pure $\mathrm{SnO}_{2}$. The band structures of $\mathrm{Sn}_{0.95} \mathrm{Co}_{0.05} \mathrm{O}_{2}$ changed a lot with SIC approximation compared with LDA approximation, the bandgaps became smaller than that of pure $\mathrm{SnO}_{2}$ for LDA approximation, and larger than $\mathrm{SnO}_{2}$ with SIC approximation (figure 6).

\section{Conclusions}

This paper describes the synthesis of $\mathrm{Sn}_{0.95} \mathrm{Co}_{0.05} \mathrm{O}_{2}$ nanocrystalline using a co-precipitation method. XRD spectrum is in good agreement with the standard peak position of $\mathrm{SnO}_{2}$ (JCPDS card no. 82-0514), without any secondary phase. Magnetic measurement showed that the samples are ferromagnetic at room temperature, welldefined hysteresis loop at $300 \mathrm{~K}$ temperature. Based on first-principles spin-density functional calculations, using the Korringa-Kohn-Rostoker method (KKR) combined with the coherent potential approximation (CPA). The ferromagnetic state energy was calculated and compared with the local-moment-disordered (LMD) state energy for LDA (local density approximation) and LDA-SIC approximation. Mechanism of hybridization and interaction between magnetic ions in $\mathrm{Sn}_{0.95} \mathrm{Co}_{0.05} \mathrm{O}_{2}$ are also investigated.

\section{References}

Akai H http://sham.phys.sci.osaka-u.ac.jp/ kkr/

Bagheri MM, Mohagheghi and Shokooh-Saremi M 2010 Physica B405 4205

Belghazi Y, Schmerber G, Colis S, Rehspringer J L, Berrada A and Dinia A 2006 Appl. Phys. Lett. 89122504

Cho Y M, Choo W K, Kim H, Kim D and Ihm Y E 2002 Appl. Phys. Lett. 803358

Coey J M D, Douvalis A P, Fitzgerald C B and Venkatesan M 2004 Appl. Phys. Lett. 841332

Dielt T, Ohno H, Matsukura F, Cibert J and Ferrand D 2000 Science 2871019

Fang L M, Zu X T, Li Z J, Liu C M, Wang L M and Gao F 2008 J. Mater. Sci. Mater. Electron. 19868 
Fitzgerald C B, Venkatesan M, Douvalais A P, Huber S, Coey J M D and Bakas T 2004 Appl. Phys. Lett. 957390

Fitzgerald C B et al 2006 Phys. Rev. B74 115307

Han S J et al 2002 Appl. Phys. Lett. 814212

Hidalgo P, Castro R H R, Coelho A C V and Gouvea D 2005 Chem. Mater. 174149

Hong N H and J Sakai 2005 Physica B358 265

Hong N H, Ruyter A, Prellier W, Sakai, J. and Huong N T 2005 J. Phys.: Condens. Matter 176533

Hong N H, Sakai J, Prellier W and Hassini A 2005 J. Phys.: Condens. Matter 171697

Lalauze R, Le Theisse J C, Pijolat C and Soustelle M 1987 Solid State Ionics 12453

Liu S J, Liu C Y, Juang J Y and Fang H W 2009 J. Appl. Phys. 105013928

Matsumoto Y et al 2001 Science 291854

Mounkachi O et al 2012 J. Magn. Magn. Mater. 3241945

Ogale S B et al 2003 Phys. Rev. Lett. 91077205

Ohno H 1998 Science 281951
Pal B and Gir P K 2011 J. Nanosci. Nanotechnol. 111

Prinz G A 1998 Science 2821660

Punnoose A et al 2005 Phys. Rev. B72 054402

Rahman G, Garćia-Súarez V M and Hong S C 2008 Phys. Rev. B78 184404

Saeki H, Tabata and Kawai T 2001 Solid State Commun. 120 439

Srinivas K, Vithal M, Sreedhar B, Manivel Raja M and Venugopal Reddy P 2009 J. Phys. Chem. C113 3543

Vishwakarma S R, Rahmatullah and Prasad H C 1993 J. Phys. D26 959

Vosko S H, Wilk L and Nusair M 1980 Can. J. Phys. 58 1200

Wang X L, Dai Z X, Zeng 2008 J. Phys.: Condens. Matter 20 045214

Wang X L, Zeng Z, Zheng X H and Lin H Q 2007 J. Appl. Phys. 101 09H104

Wu S Y et al 2003 Appl. Phys. Lett. 823047

Zhang C W and Yan S S 2009 J. Appl. Phys. 106063709 